\title{
Identification of putative drugs for gastric adenocarcinoma utilizing differentially expressed genes and connectivity map
}

\author{
ZU-XUAN CHEN ${ }^{1}$, XIAO-PING ZOU ${ }^{2}$, HUANG-QUN YAN $^{2}$, RUI ZHANG ${ }^{2}$, JIN-SHU PANG ${ }^{2}$, \\ XIN-GAN QIN ${ }^{3}$, RONG-QUAN HE ${ }^{4}$, JIE MA ${ }^{4}$, ZHEN-BO FENG $^{2}$, GANG CHEN $^{2}$ and TING-QING GAN ${ }^{1}$ \\ ${ }^{1}$ Department of Medical Oncology, The Second Affiliated Hospital of Guangxi Medical University; \\ Departments of ${ }^{2}$ Pathology, ${ }^{3}$ Gastrointestinal Surgery and ${ }^{4}$ Medical Oncology, \\ The First Affiliated Hospital of Guangxi Medical University, Nanning, \\ Guangxi Zhuang Autonomous Region 530021, P.R. China
}

Received April 19, 2018; Accepted November 20, 2018

DOI: $10.3892 / \mathrm{mmr} .2018 .9758$

\begin{abstract}
Gastric adenocarcinoma (GAC) is a challenging disease with dim prognosis even after surgery; hence, novel treatments for GAC are in urgent need. The aim of the present study was to explore new potential compounds interfering with the key pathways related to GAC progression. The differentially expressed genes (DEGs) between GAC and adjacent tissues were identified from The Cancer Genome Atlas (TCGA) and Genotype-Tissue Expression (GTEx) database. Connectivity Map (CMap) was performed to screen candidate compounds for treating GAC. Subsequently, pathways affected by compounds were overlapped with those enriched by the DEGs to further identify compounds which had anti-GAC potential. A total of 843 DEGs of GAC were identified. Via Kyoto Encyclopedia of Genes and Genomes (KEGG) analysis, 13 pathways were significantly enriched. Moreover, 78 compounds with markedly negative correlations with DEGs were revealed in CMap database $(\mathrm{P}<0.05$ and Enrichment $<0)$. Subpathways of cell cycle and p53 signaling pathways, and core genes of these compounds, cyclin $\mathrm{B} 1$ (CCNB1) and CDC6, were identified. This study further revealed seven compounds that may be effective against GAC; in particular
\end{abstract}

Correspondence to: Professor Ting-Qing Gan, Department of Medical Oncology, The Second Affiliated Hospital of Guangxi Medical University, 166 Daxuexi Road, Nanning, Guangxi Zhuang Autonomous Region 530021, P.R. China

E-mail: gantingqing_gxmu@163.com

Abbreviations: GAC, gastric adenocarcinoma; CMap, connectivity map; TCGA, The Cancer Genome Atlas; DEGs, differentially expressed genes; KEGG, Kyoto Encyclopedia of Genes and Genomes; FC, fold change; GO, Gene Ontology; PPI, protein-protein interaction; HD, Hirschsprung disease; NAG, non-steroidal anti-inflammatory drug-activated gene; FOXM1, forkhead box transcription factor 1

Key words: differentially expressed genes, pathways, connectivity map, gastric adenocarcinoma methylbenzethonium chloride and alexidine have never yet been reported for GAC treatment. In brief, the candidate drugs identified in this study may provide new options to improve the treatment of patients with GAC. However, the biological effects of these drugs need further investigation.

\section{Introduction}

Globally, gastric cancer is the fifth leading cause of cancer and the third leading cause of death from cancer $(1,2)$. In 2015, 679,100 new cases of gastric cancer were diagnosed in China, accounting for $15.8 \%$ of the total number of newly occurred cancer cases. In addition, gastric cancer resulted in 498,000 deaths, $17.7 \%$ of all cancer-related deaths, and the incidence of gastric cancer has been steadily increasing (3). Among these cases, gastric adenocarcinoma (GAC) accounts for $95 \%$ of all gastric cancer cases. Research indicates that, even after surgery, the outcome of GAC patients remains dim (4-6). Therefore, other novel treatments for GAC should be developed. The study of small-molecule drugs aiming at multiple protein pathways modulating tumor progression, invasion, and metastasis formation, has received much interest in recent years (7-9). The purpose of this study was to discover new, potential small-molecule drugs by using multiple online databases.

Connectivity Map (CMap) is one of the gene expression profile databases used to process the genetic data. CMap was developed by Lamb and his colleagues from Broad Institute of MIT, Whitehead Institute and Harvard Medical School, (Boston, MA, USA) (10). CMap utilizes the differential gene expression of human cells which are treated with small-molecule drugs, to construct a biological application database based on connection of small-molecule drugs, gene expression and different diseases. CMap allows scholars of drug development to take advantage of gene expression profiling data and, therefore, identify the drugs highly correlated with disease, infer the main chemical structure of most drug molecules, and summarize the mechanism of possible action of drug molecules.

To explore new drugs for GAC, based on the integrated subpathway analysis, we implemented an in silico method 
for the reuse of GAC drugs. First, we identified the differentially expressed genes (DEGs) between GAC and non-tumor tissues identified in The Cancer Genome Atlas (TCGA) and Genotype-Tissue Expression (GTEx) databases, and then determined the potential pathways affecting the progression of GAC. Next, CMap was used to verify the pathways of GAC affected by small-molecule treatment. Finally, small-molecule drugs that can target subpathways related to GAC were considered as potential new agents in the treatment of GAC (Fig. 1). The candidate drugs identified in our approach may provide a new direction for improving the treatment of patients with GAC.

\section{Materials and methods}

$D E G$ analysis of GAC. Using the GEPIA online analysis website (http://gepia.cancer-pku.cn/), the expression data of mRNA of GAC in TCGA and GTEx databases were performed with the value of fold change (FC). Among these data, only the genes with $\log \mathrm{FC}>2$ and $\log \mathrm{FC}<-2$ were defined as DEGs, including upregulated and downregulated ones.

Enrichment analysis of DEGs. DEGs were performed with Gene Ontology (GO) and the Kyoto Encyclopedia of Genes and Genomes (KEGG) pathway analysis with the WebGestalt database (http://www.webgestalt.org/). Also, pathway analysis was conducted by Gene List Analysis (http://www.pantherdb. org/) to obtain possible pathways during the development of GAC. Finally, we used the STRING database (https://string-db. org/) to analyze the protein-protein interaction (PPI) of the ultimate DEGs as previously reported (11-16). In this study, GO outcomes were analyzed visibly with Cytoscape software (version 3.7.0, U.S. National Institute of General Medical Sciences (NIGMS), https://cytoscape.org/).

CMap for DEG analysis of drug molecule cures for GAC. The CMap database (https://portals.broadinstitute.org/CMap/) (build 02) contains over 7,000 gene expression profiles and 1,309 chemicals. To analyze this potential mechanism for the development of GAC, we first set up the files in query signature format for DEGs obtained from the TCGA (https://cancergenome.nih.gov/) and GTEx databases (https://gtexportal.org/home/). We then entered the CMap quick query interface to import the files of upregulated and downregulated genes and ran them with CMap analysis. In this way, we analyzed the drug molecules for the DEGs of GAC (17). The negatively related drugs $(\mathrm{P}<0.05$ and Enrichment $<0$ ) for anti-GAC were then screened.

Correlation data between drug molecules and subpathways. The chip expression profiles of 1,309 drugs and the genes affected by the drugs using the CMap database were downloaded. Furthermore, we identified the subpathways that obtain significant enrichment for each small-molecule drug with the affected genes according to the method reported by a previous publication (18). Consistent with the reference, 196 small-molecular drugs and 104 subpathways were also achieved. The overlapped pathways between those from CMap and those enriched by DEGs were determined, which were identified as potential pathways related to both the treatment

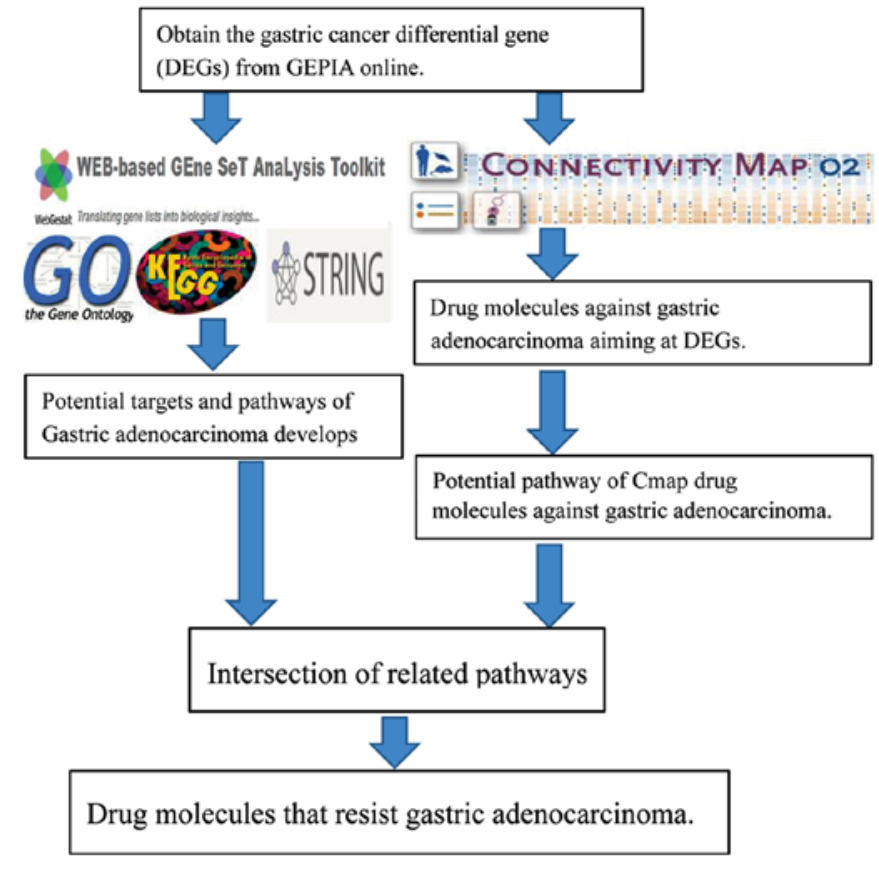

Figure 1. The flow chart of the study process.

and pathogenesis of GAC. Finally, the drug-pathway network was constructed for GAC.

\section{Results}

Screening results of DEGs. Altogether, 843 DEGs in mRNA expression of GAC were obtained, which included 638 upregulated genes and 205 downregulated ones. The next analysis was based on this screening result.

Functional annotation, pathway enrichment and PPI network analysis. Through GO analysis, in the annotations of biological progress, the top three most significant processes were mitotic sister chromatid segregation, mitotic cell cycle and nuclear division. In the terms of cellular component, the top most significant annotations were extracellular space, chromosome, centromeric region and spindle. As for analysis of molecular function, the top three most significant functions were serine hydrolase activity, chemokine activity and serine-type peptidase activity (Table I and Fig. 2). KEGG pathway analysis indicated that DEGs were obviously centralized in 13 pathways, including cell cycle, protein digestion and absorption, Staphylococcus aureus infection, and the p53 signaling pathway (Table II and Fig. 3). From the PPI network analysis, we acquired the following hub genes: $C C N B 1$, AURKA, CDC6, KIF11, OIP5, NCAPG, KIF23, DLGAP5 and NDC80 (nodes $\geq 100$ ) (Fig. 4).

CMap analysis to achieve potential compounds for GAC. The 843 DEGs of GAC mentioned above led to 78 compounds by CMap (Table III) when $\mathrm{P}<0.05$ and Enrichment $<0$.

Intersection of small-molecule drug correlative pathways and KEGG pathways. According to a previous method (18), we performed subpathway analysis and obtained 
Table I. Top 10 of the most significantly enriched GO terms.

\begin{tabular}{|c|c|c|c|c|}
\hline Pathway ID & Terms & Gene count & FDR & P-value \\
\hline \multicolumn{5}{|l|}{$\mathrm{BP}$} \\
\hline GO:0000070 & Mitotic sister chromatid segregation & 36 & 0 & 0 \\
\hline GO:0000278 & Mitotic cell cycle & 112 & 0 & 0 \\
\hline GO:0000280 & Nuclear division & 76 & 0 & 0 \\
\hline GO:0000819 & Sister chromatid segregation & 47 & 0 & 0 \\
\hline GO:0007049 & Cell cycle & 142 & 0 & 0 \\
\hline GO:0007059 & Chromosome segregation & 58 & 0 & 0 \\
\hline GO:0007067 & Mitotic nuclear division & 69 & 0 & 0 \\
\hline GO:0008283 & Cell proliferation & 165 & 0 & 0 \\
\hline GO:0022402 & Cell cycle process & 125 & 0 & 0 \\
\hline GO:0042127 & Regulation of cell proliferation & 127 & 0 & 0 \\
\hline \multicolumn{5}{|l|}{$\mathrm{CC}$} \\
\hline GO:0005615 & Extracellular space & 129 & 0 & 0 \\
\hline GO:0000775 & Chromosome, centromeric region & 34 & $1.14 \mathrm{E}-13$ & $2.22 \mathrm{E}-16$ \\
\hline GO:0005819 & Spindle & 42 & $1.52 \mathrm{E}-13$ & $4.44 \mathrm{E}-16$ \\
\hline GO:0000793 & Condensed chromosome & 35 & $2.86 \mathrm{E}-13$ & $1.11 \mathrm{E}-15$ \\
\hline GO:0098687 & Chromosomal region & 42 & $2.54 \mathrm{E}-12$ & $1.23 \mathrm{E}-14$ \\
\hline GO:0000779 & Condensed chromosome, centromeric region & 25 & $2.88 \mathrm{E}-12$ & $1.68 \mathrm{E}-14$ \\
\hline GO:0000776 & Kinetochore & 26 & $1.18 \mathrm{E}-11$ & $8.04 \mathrm{E}-14$ \\
\hline GO:0000777 & Condensed chromosome kinetochore & 23 & $1.39 \mathrm{E}-11$ & $1.20 \mathrm{E}-13$ \\
\hline GO:0009986 & Cell surface & 64 & $1.39 \mathrm{E}-11$ & $1.21 \mathrm{E}-13$ \\
\hline GO:0005694 & Chromosome & 71 & $1.40 \mathrm{E}-10$ & $1.36 \mathrm{E}-12$ \\
\hline \multicolumn{5}{|l|}{ MF } \\
\hline GO:0017171 & Serine hydrolase activity & 30 & $2.76 \mathrm{E}-07$ & $1.77 \mathrm{E}-10$ \\
\hline GO:0008009 & Chemokine activity & 14 & $2.76 \mathrm{E}-07$ & $3.88 \mathrm{E}-10$ \\
\hline GO:0008236 & Serine-type peptidase activity & 29 & $2.76 \mathrm{E}-07$ & $5.77 \mathrm{E}-10$ \\
\hline GO:0004252 & Serine-type endopeptidase activity & 27 & $2.76 \mathrm{E}-07$ & $6.04 \mathrm{E}-10$ \\
\hline GO:0042379 & Chemokine receptor binding & 15 & $3.08 \mathrm{E}-07$ & $8.91 \mathrm{E}-10$ \\
\hline GO:0004175 & Endopeptidase activity & 42 & $3.08 \mathrm{E}-07$ & $1.01 \mathrm{E}-09$ \\
\hline GO:0045236 & CXCR chemokine receptor binding & 9 & 4.33E-07 & $1.66 \mathrm{E}-09$ \\
\hline GO:0001664 & G-protein coupled receptor binding & 27 & $6.08 \mathrm{E}-05$ & $2.66 \mathrm{E}-07$ \\
\hline GO:0032395 & MHC class II receptor activity & 6 & $7.59 \mathrm{E}-05$ & $3.74 \mathrm{E}-07$ \\
\hline GO:0042802 & Identical protein binding & 82 & $1.18 \mathrm{E}-04$ & $6.44 \mathrm{E}-07$ \\
\hline
\end{tabular}

GO, Gene Ontology; BP, biological progress; CC, cellular component; MF, molecular function; FDR, false discovery rate.

104 subpathways. After integrating these 104 subpathways with 13 KEGG pathways generated by the DEGs, two pathways related to anti-GAC drug molecules were finally achieved (Table IV and Fig. 5), including cell cycle and p53 signaling pathways. These two pathways were related to 32 genes and seven CMap small-molecule drugs. The genes involved in these two KEGG pathway were $C D K N 2 A, D B F 4, C H E K 1$, ORC6, SFN, MAD2L1, MCM2, MCM4, MCM5, PCNA, PLK1, CCND1, BUB1, BUB1B, TTK, CDC45, CCNA2, CCNB1, PKMYT1, CCNB2, PTTG1, ESPL1, CDK1, CDC6, CDC20, CDC25C, IGFBP3, GTSE1, SERPINB5, RPRM, RRM2 and $B I D$. The PPI analysis with the above 32 genes demonstrated two hub genes (CCNBI and CDC6). The seven CMap small-molecule drugs were troglitazone, methylbenzethonium chloride, thiostrepton, alexidine, vorinostat, methotrexate and etoposide (Fig. 6).

Expression levels of CCNB1 and CDC6 $\mathrm{mRNA}$ in GAC tissues. The expression levels of $C C N B 1$ and $C D C 6$ mRNA in GACs were queried from GEPIA database (http://gepia.cancer-pku.cn/). The results showed that the two genes were both highly expressed in GAC tissues compared to non-cancerous gastric tissues (Fig. 7).

Verification of predicting small-molecule drugs of GAC with online literature retrieval. Using PubMed, we identified studies that investigated the effect of relevant drugs on GAC. We found 268 articles related to the effect of methotrexate on 
Table II. Significantly enriched KEGG pathway.

\begin{tabular}{|c|c|c|c|c|}
\hline Pathway ID & Terms & Gene count & FDR & P-value \\
\hline hsa04110 & Cell cycle & 26 & $2.83 \mathrm{E}-08$ & $9.34 \mathrm{E}-11$ \\
\hline hsa04974 & Protein digestion and absorption & 17 & $1.33 \mathrm{E}-04$ & 8.80E-07 \\
\hline hsa05150 & Staphylococcus aureus infection & 12 & $9.58 \mathrm{E}-04$ & $9.49 \mathrm{E}-06$ \\
\hline hsa04115 & p53 signaling pathway & 13 & $1.35 \mathrm{E}-03$ & $1.79 \mathrm{E}-05$ \\
\hline hsa05140 & Leishmaniasis & 12 & $9.11 \mathrm{E}-03$ & $1.50 \mathrm{E}-04$ \\
\hline hsa05323 & Rheumatoid arthritis & 13 & $1.40 \mathrm{E}-02$ & 3.07E-04 \\
\hline hsa04610 & Complement and coagulation cascades & 12 & $1.40 \mathrm{E}-02$ & 3.24E-04 \\
\hline hsa05416 & Viral myocarditis & 10 & $1.56 \mathrm{E}-02$ & 4.13E-04 \\
\hline hsa05310 & Asthma & 7 & $1.73 \mathrm{E}-02$ & $5.12 \mathrm{E}-04$ \\
\hline hsa05164 & Influenza A & 18 & 4.47E-02 & $1.63 \mathrm{E}-03$ \\
\hline hsa04512 & ECM-receptor interaction & 11 & 4.47E-02 & 1.64E-03 \\
\hline hsa04060 & Cytokine-cytokine receptor interaction & 24 & 4.47E-02 & $1.77 \mathrm{E}-03$ \\
\hline hsa04640 & Hematopoietic cell lineage & 12 & $4.86 \mathrm{E}-02$ & 2.09E-03 \\
\hline
\end{tabular}

KEGG, Kyoto Encyclopedia of Genes and Genomes; FDR, false discovery rate.

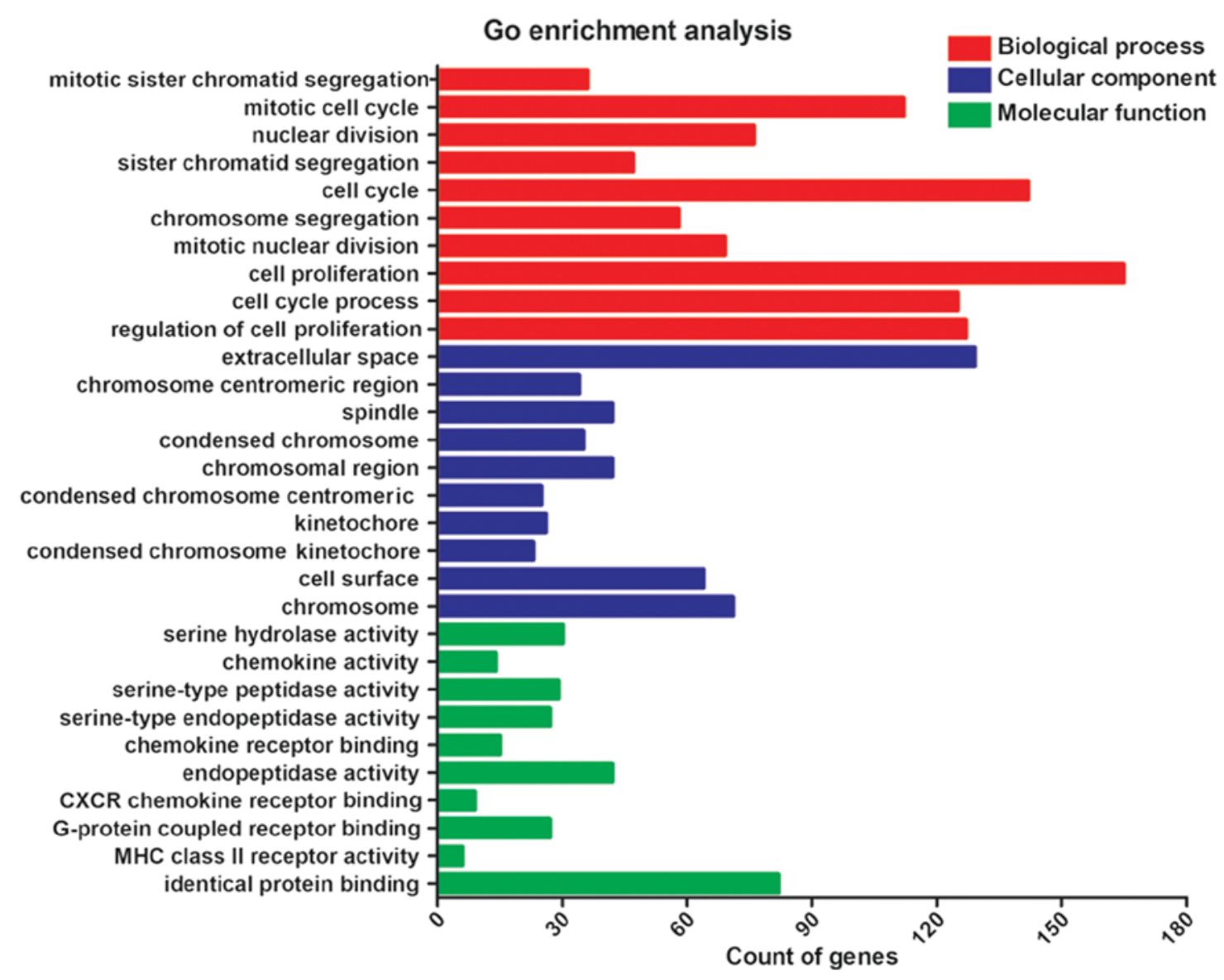

Figure 2. Gene Ontology (GO) enrichment analysis of the differentially expressed genes in gastric adenocarcinoma.

GAC, 403 articles related to etoposide, and 17 articles related to troglitazone, which is a diabetes drug that may inhibit GAC. Nine studies concerned vorinostat and three studies were related to thiostrepton. Most importantly, methylbenzethonium chloride and alexidine have never been addressed in the literature of GAC.

\section{Discussion}

In the present study, we identified DEGs of GAC and found several pathways and hub genes that may play a critical role in the pathogenesis and development of GAC. Also, through 


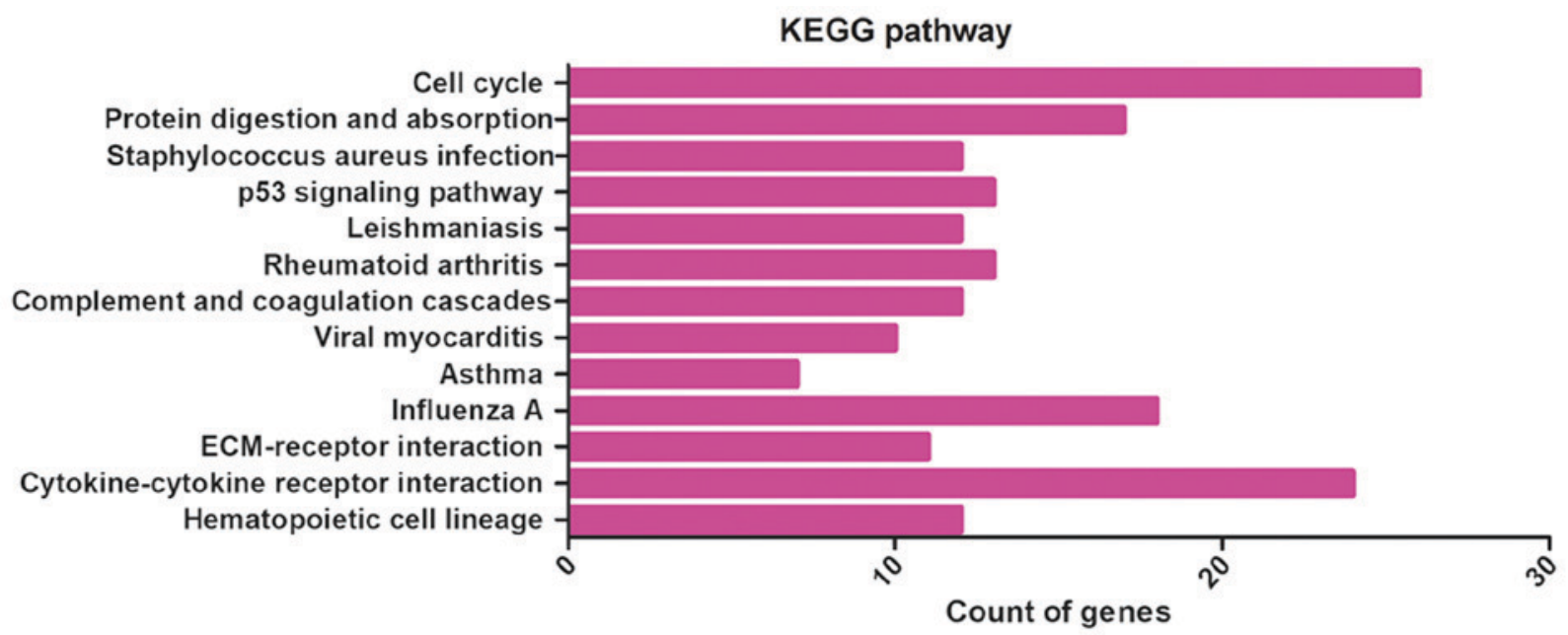

Figure 3. Kyoto Encyclopedia of Genes and Genomes (KEGG) pathway analysis of the differentially expressed genes in gastric adenocarcinoma.

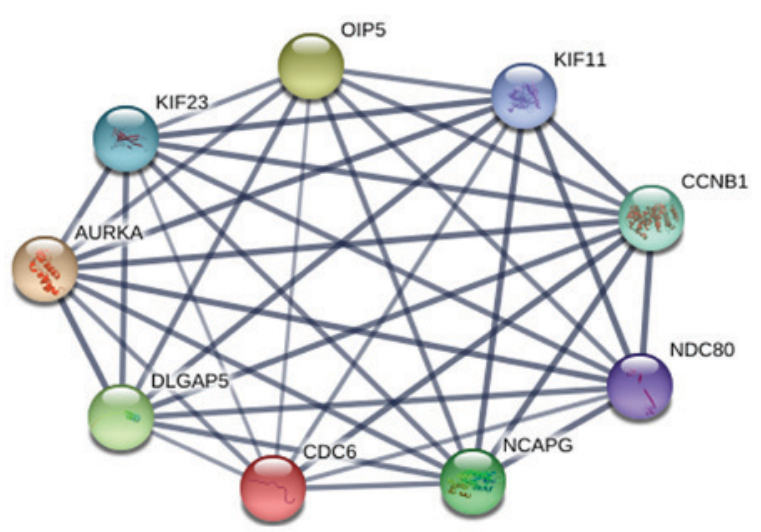

Figure 4. The top 9 hub genes with most interaction lines in protein-protein interaction (PPI) analysis.

the connectivity mapping approach, some known compounds were found to share similar pathways of those generated from the DEGs of GAC, including methotrexate, etoposide, troglitazone, thiostrepton, vorinostat, methylbenzethonium chloride and alexidine. The findings from the present study suggest that methylbenzethonium chloride and alexidine could act as novel potential drugs for the treatment of GAC and warrant further investigation, as they have never been tested previously.

The CMap database reveals the connection between disease, genes and drugs, using gene expression data and the 'similarity' concept with a small-molecular compound or the gene expression spectrum of the drug as the core (19). CMap database provides a unique method for drug development through comparison to filter candidate compounds curing diseases, and it has been adopted by several scholars $(20,21)$. For instance, Xiao et al used gene expression profile chip technology and the CMap database to study molecular mechanisms of Hirschsprung disease (HD) and potential drugs. They found differences in the neuronal developmental disorders of HD genes and signaling pathways, and discovered that some compounds may offset the damage of HD development (22).

In this study, the DEGs between GAC and adjacent tissues were compared with the expression profiles in CMap to identify negatively correlative compounds that are potential compounds for GAC. Among the candidate compounds determined in the present study, two compounds (alexidine and methylbenzethonium) are particularly important. Alexidine is an antimicrobial agent with high affinity for bacteria, which can be used in the root canal irrigation solution of oral treatment (23). Feng et al, using high-throughput drug screening tests, identified that alexidine is an antitumor drug that can inhibit cytokines and growth factors necessary for multiple myeloma (24). Meanwhile, methylbenzethonium chloride, a broad spectrum antibiotic, was found to be able to specifically induce apoptosis in undifferentiated embryonic stem cells of mice (25). The effect could be applied to prevent reoccurrence of the tumor after stem cell transplantation therapy. Methylbenzethonium chloride may become another novel anticancer agent (25).

The present study showed that alexidine had the lowest connectivity score (-0.996), indicating a highly negative correlation with the DEGs of GAC. The connectivity score of methylbenzethonium chloride also suggests that it has the capacity to inhibit the growth of GAC. In addition, this study predicted that both alexidine and methylbenzethonium chloride can play a vital role in inhibiting GAC by regulating the p53 signaling pathway. Previous studies have shown that the p53 signaling pathway regulates various cellular functions, including apoptosis, induction of aging, and inhibition of cell growth, migration and invasion (26-28). However, the specific molecular mechanisms of alexidine and methylbenzethonium chloride for antitumor activity need to be further explored.

Five other compounds achieved in the present study have been mentioned in other studies. Troglitazone hinders BGC-823 GAC cell proliferation and promotes its apoptosis by inducing expression of the non-steroidal anti-inflammatory drug-activated gene (NAG) (29). In addition, thiostrepton was found to reverse drug resistance in GAC by inhibiting the forkhead box transcription factor 1 (FOXM1) (30). Vorinostat (31), methotrexate (32) and etoposide (33) are proven to inhibit the proliferation of GAC cells. This evidence indicates that the predictive method in this study is convincing and worth being used for drug exploration. 
Table III. CMap compounds matched by the DEGs of gastric adenocarcinoma.

\begin{tabular}{|c|c|c|c|c|c|c|c|}
\hline Rank & CMap name & Cell line & $\mathrm{N}$ & Enrichment & P-value & Specificity & Percent non-null \\
\hline 1 & Phenoxybenzamine & MCF7 & 3 & -0.984 & 0 & 0 & 100 \\
\hline 2 & Vorinostat & MCF7 & 7 & -0.844 & 0 & 0.1262 & 100 \\
\hline 3 & Trichostatin A & PC3 & 55 & -0.705 & 0 & 0.1149 & 96 \\
\hline 4 & Trichostatin A & MCF7 & 92 & -0.59 & 0 & 0.1881 & 88 \\
\hline 5 & Trichostatin A & HL60 & 34 & -0.465 & 0 & 0.1946 & 52 \\
\hline 6 & LY-294002 & MCF7 & 34 & -0.454 & 0 & 0.1625 & 70 \\
\hline 7 & Resveratrol & MCF7 & 6 & -0.865 & 0.00002 & 0.0082 & 100 \\
\hline 8 & Alexidine & PC3 & 2 & -0.996 & 0.00004 & 0 & 100 \\
\hline 9 & 15-Delta prostaglandin $\mathrm{J} 2$ & MCF7 & 8 & -0.695 & 0.00018 & 0.0414 & 87 \\
\hline 10 & Meticrane & PC3 & 2 & -0.991 & 0.00026 & 0 & 100 \\
\hline 11 & Astemizole & PC3 & 2 & -0.99 & 0.00026 & 0.0192 & 100 \\
\hline 12 & Thiostrepton & MCF7 & 2 & -0.973 & 0.00141 & 0.0283 & 100 \\
\hline 13 & Clemizole & PC3 & 2 & -0.973 & 0.00141 & 0 & 100 \\
\hline 14 & Sulconazole & MCF7 & 2 & -0.973 & 0.00157 & 0 & 100 \\
\hline 15 & Mefloquine & PC3 & 2 & -0.971 & 0.00167 & 0.0431 & 100 \\
\hline 16 & MG-262 & PC3 & 2 & -0.968 & 0.00223 & 0.0738 & 100 \\
\hline 17 & Cloperastine & PC3 & 2 & -0.968 & 0.00223 & 0.0149 & 100 \\
\hline 18 & Thioridazine & PC3 & 5 & -0.736 & 0.0027 & 0.102 & 100 \\
\hline 19 & Methotrexate & MCF7 & 3 & -0.877 & 0.00379 & 0.0853 & 100 \\
\hline 20 & Valproic acid & HL60 & 14 & -0.448 & 0.00403 & 0.2883 & 64 \\
\hline 21 & Cloperastine & MCF7 & 3 & -0.873 & 0.00415 & 0.0196 & 100 \\
\hline 22 & Fludroxycortide & PC3 & 2 & -0.954 & 0.00453 & 0.0171 & 100 \\
\hline 23 & Pyrantel & PC3 & 2 & -0.946 & 0.00644 & 0.0144 & 100 \\
\hline 24 & Thioguanosine & MCF7 & 2 & -0.945 & 0.00658 & 0.0455 & 100 \\
\hline 25 & $\begin{array}{l}\text { 6-Bromoindirubin-3'-oxime } \\
\text { methylbenzethonium }\end{array}$ & PC3 & 4 & -0.755 & 0.00732 & 0.0498 & 100 \\
\hline 26 & Chloride & PC3 & 2 & -0.939 & 0.00767 & 0.0598 & 100 \\
\hline 27 & Chlorpromazine & PC3 & 4 & -0.749 & 0.0079 & 0.0168 & 100 \\
\hline 28 & Vorinostat & HL60 & 3 & -0.839 & 0.00837 & 0.1705 & 100 \\
\hline 29 & Vitexin & MCF7 & 2 & -0.936 & 0.00861 & 0.0051 & 100 \\
\hline 30 & Acetazolamide & MCF7 & 2 & -0.931 & 0.00984 & 0 & 100 \\
\hline 31 & Pyrvinium & MCF7 & 4 & -0.731 & 0.0105 & 0.1304 & 100 \\
\hline 32 & 5224221 & MCF7 & 2 & -0.927 & 0.01097 & 0.1429 & 100 \\
\hline 33 & Methacholine chloride & MCF7 & 2 & -0.924 & 0.01181 & 0.0278 & 100 \\
\hline 34 & Cortisone & MCF7 & 2 & -0.921 & 0.01262 & 0.0117 & 100 \\
\hline 35 & Carbachol & MCF7 & 2 & -0.919 & 0.01318 & 0.0058 & 100 \\
\hline 36 & Clotrimazole & MCF7 & 3 & -0.807 & 0.01444 & 0.0556 & 100 \\
\hline 37 & Dipyridamole & MCF7 & 3 & -0.799 & 0.01671 & 0.04 & 100 \\
\hline 38 & Abamectin & MCF7 & 2 & -0.907 & 0.01746 & 0.05 & 100 \\
\hline 39 & LY-294002 & PC3 & 12 & -0.423 & 0.01802 & 0.3669 & 66 \\
\hline 40 & Troglitazone & PC3 & 4 & -0.696 & 0.01804 & 0.1159 & 100 \\
\hline 41 & Luteolin & MCF7 & 2 & -0.904 & 0.01839 & 0.0476 & 100 \\
\hline 42 & Hydroflumethiazide & MCF7 & 2 & -0.902 & 0.01913 & 0.0601 & 100 \\
\hline 43 & Homochlorcyclizine & MCF7 & 2 & -0.898 & 0.02066 & 0.0968 & 100 \\
\hline 44 & Gemfibrozil & PC3 & 2 & -0.896 & 0.02167 & 0.0208 & 100 \\
\hline 45 & Withaferin A & PC3 & 2 & -0.894 & 0.02223 & 0.0917 & 100 \\
\hline 46 & Tanespimycin & PC3 & 12 & -0.414 & 0.02239 & 0.3382 & 58 \\
\hline 47 & Prochlorperazine & MCF7 & 9 & -0.472 & 0.0231 & 0.1892 & 66 \\
\hline 48 & Ciclosporin & MCF7 & 4 & -0.679 & 0.02349 & 0.0576 & 75 \\
\hline 49 & Disulfiram & PC3 & 2 & -0.891 & 0.02382 & 0.0667 & 100 \\
\hline 50 & Procaine & PC3 & 2 & -0.89 & 0.024 & 0.0294 & 100 \\
\hline 51 & 0173570-0000 & PC3 & 4 & -0.677 & 0.02407 & 0.1349 & 75 \\
\hline
\end{tabular}


Table III. Continued.

\begin{tabular}{|c|c|c|c|c|c|c|c|}
\hline Rank & CMap name & Cell line & $\mathrm{N}$ & Enrichment & P-value & Specificity & Percent non-null \\
\hline 52 & Tretinoin & MCF7 & 13 & -0.395 & 0.02531 & 0.3655 & 61 \\
\hline 53 & Fluphenazine & PC3 & 3 & -0.769 & 0.02534 & 0.1026 & 100 \\
\hline 54 & Loperamide & MCF7 & 3 & -0.767 & 0.026 & 0.087 & 100 \\
\hline 55 & Dilazep & PC3 & 2 & -0.886 & 0.02612 & 0.0784 & 100 \\
\hline 56 & Trifluoperazine & PC3 & 3 & -0.765 & 0.02656 & 0.1379 & 100 \\
\hline 57 & 3-Acetylcoumarin & MCF7 & 3 & -0.764 & 0.02692 & 0.022 & 100 \\
\hline 58 & Flunarizine & MCF7 & 2 & -0.884 & 0.02712 & 0.068 & 100 \\
\hline 59 & Sulfaguanidine & PC3 & 2 & -0.878 & 0.02972 & 0.0202 & 100 \\
\hline 60 & Ethaverine & MCF7 & 2 & -0.878 & 0.03004 & 0.0133 & 100 \\
\hline 61 & Amiodarone & MCF7 & 3 & -0.754 & 0.03043 & 0.1039 & 100 \\
\hline 62 & Picotamide & PC3 & 2 & -0.875 & 0.03127 & 0.0162 & 100 \\
\hline 63 & Felodipine & MCF7 & 5 & -0.594 & 0.0318 & 0.1376 & 80 \\
\hline 64 & Prestwick-1084 & MCF7 & 2 & -0.873 & 0.03201 & 0.0545 & 100 \\
\hline 65 & Monobenzone & MCF7 & 2 & -0.871 & 0.03306 & 0.0548 & 100 \\
\hline 66 & Pioglitazone & PC3 & 5 & -0.586 & 0.03585 & 0.3436 & 60 \\
\hline 67 & Levocabastine & MCF7 & 2 & -0.866 & 0.03626 & 0.0615 & 100 \\
\hline 68 & Noretynodrel & MCF7 & 2 & -0.865 & 0.03628 & 0.0822 & 100 \\
\hline 69 & Trifluoperazine & MCF7 & 9 & -0.448 & 0.03655 & 0.2308 & 55 \\
\hline 70 & 15-Delta prostaglandin $\mathrm{J} 2$ & HL60 & 3 & -0.738 & 0.03684 & 0.1429 & 100 \\
\hline 71 & Etoposide & MCF7 & 2 & -0.864 & 0.03712 & 0.1 & 100 \\
\hline 72 & Bufexamac & MCF7 & 2 & -0.863 & 0.0376 & 0.0556 & 100 \\
\hline 73 & 0179445-0000 & PC3 & 4 & -0.644 & 0.03853 & 0.0685 & 75 \\
\hline 74 & 15-Delta prostaglandin $\mathrm{J} 2$ & PC3 & 3 & -0.734 & 0.03856 & 0.1507 & 100 \\
\hline 75 & Minaprine & PC3 & 2 & -0.858 & 0.04008 & 0.031 & 100 \\
\hline 76 & Oxymetazoline & PC3 & 2 & -0.855 & 0.04181 & 0.0345 & 100 \\
\hline 77 & Nortriptyline & MCF7 & 2 & -0.852 & 0.04338 & 0.0901 & 100 \\
\hline 78 & CP-690334-01 & MCF7 & 4 & -0.633 & 0.04418 & 0.1027 & 50 \\
\hline 79 & SB-203580 & PC3 & 2 & -0.85 & 0.04515 & 0.0464 & 100 \\
\hline 80 & Scriptaid & PC3 & 2 & -0.849 & 0.04537 & 0.1596 & 100 \\
\hline 81 & Esculetin & MCF7 & 2 & -0.848 & 0.04609 & 0.0671 & 100 \\
\hline 82 & Fluspirilene & MCF7 & 2 & -0.848 & 0.0464 & 0.1748 & 100 \\
\hline 83 & Sulfadoxine & MCF7 & 2 & -0.845 & 0.04829 & 0.0481 & 100 \\
\hline 84 & Monorden & PC3 & 5 & -0.562 & 0.04932 & 0.106 & 60 \\
\hline 85 & Ivermectin & MCF7 & 2 & -0.843 & 0.04937 & 0.1404 & 100 \\
\hline 86 & Norethisterone & MCF7 & 2 & -0.842 & 0.04994 & 0.0263 & 100 \\
\hline
\end{tabular}

CMap, Connectivity Map; DEGs, differentially expressed genes. N, number of all instances of the same perturbagen made in the same cell line. A total of 78 compounds were included, among which, four compounds were administered to two different cell lines and two compounds were administered to three different cell lines. Thus, there are 86 rows in the table.

In this study, we used bioinformatic methods to screen differentially expressing potential genetic biomarkers based on RNA-seq data. The results of pathway enrichment analysis indicated 13 pathways which were evidently enriched with DEGs, including the cell cycle, protein digestion and absorption, Staphylococcus aureus infection and the p53 signaling pathway. In addition, these DEGs were analyzed with CMap and subpathways, and two (cell cycle and p53 signaling pathway) were found to be closely related to the treatment potential and occurrence of GAC. CCNB1 and CDC6 in these pathways were also hub genes in the PPI network.
The clinical role of these hub genes was analyzed also based on publicly available RNA-seq data, and it was found that CCNB1 was upregulated in patients with GAC. CCNB1 is a member of the cell cycle protein B family; it is a regulatory protein involved in mitosis, mostly expressed in the $\mathrm{G} 2 / \mathrm{M}$ period, and plays a significant role in the S-to-G2/M phases (34). Therefore, overexpression of CCNB1 in GAC leads to chaos in the cell cycle, mitosis promotion and cell proliferation. Previous research has shown that silencing of CDKN3 stimulates cell cycle arrest by reducing the expression of CDK2, CDC25, CCNB1 and CCNB2 in human GAC 
Table IV. CMap negatively correlated compounds matched by pathway.

\begin{tabular}{|c|c|c|}
\hline Drug name & Pathway name & Subpathway ID \\
\hline Alexidine & p53 signaling pathway & path:04115_2; path:04115_1; path:04115_7 \\
\hline Mefloquine & Toll-like receptor signaling pathway & $\begin{array}{l}\text { path:04620_17; path:04620_18; path:04620_22; } \\
\text { path:04620_9 }\end{array}$ \\
\hline Mefloquine & Steroid hormone biosynthesis & $\begin{array}{l}\text { path:00140_3; path:00140_19; path:00140_16; } \\
\text { path:00140_8 }\end{array}$ \\
\hline Astemizole & Toll-like receptor signaling pathway & $\begin{array}{l}\text { path:04620_12; path:04620_9; path:04620_18; } \\
\text { path:04620_17 }\end{array}$ \\
\hline Thiostrepton & p53 signaling pathway & path:04115_1 \\
\hline Methotrexate & p53 signaling pathway & $\begin{array}{l}\text { path:04115_7; path:04115_1; path:04115_4; } \\
\text { path:04115_3; path:04115_2 }\end{array}$ \\
\hline Sulconazole & Metabolism of xenobiotics by cytochrome P450 & path:00980_3 \\
\hline Resveratrol & Tryptophan metabolism & path:00380_5 \\
\hline Resveratrol & Toxoplasmosis & path:05145_18 \\
\hline Thioguanosine & Steroid hormone biosynthesis & path:00140_7; path:00140_8 \\
\hline MG-262 & Steroid hormone biosynthesis & $\begin{array}{l}\text { path:00140_1; path:00140_9; path:00140_8; } \\
\text { path:00140_6; path:00140_5 }\end{array}$ \\
\hline $\begin{array}{l}\text { Methylbenzethonium } \\
\text { chloride }\end{array}$ & p53 signaling pathway & path:04115_1 \\
\hline Monobenzone & MAPK signaling pathway & path:04010_30 \\
\hline Trifluoperazine & Protein processing in endoplasmic reticulum & path:04141_18: path:04141_1 \\
\hline 5224221 & Steroid hormone biosynthesis & $\begin{array}{l}\text { path:00140_18; path:00140_27; path:00140_9; } \\
\text { path:00140_8; path:00140_4 }\end{array}$ \\
\hline Vitexin & Steroid hormone biosynthesis & path:00140_19 \\
\hline Disulfiram & Protein processing in endoplasmic reticulum & path:04141_1 \\
\hline Thioridazine & Pathways in cancer & path:05200_29; path:05200_18; path:05200_11 \\
\hline Vorinostat & p53 signaling pathway & $\begin{array}{l}\text { path:04115_1; path:04115_2; path:04115_4; } \\
\text { path:04115_3 }\end{array}$ \\
\hline Etoposide & p53 signaling pathway & path:04115_7; path:04115_1; path:04115_3 \\
\hline Withaferin A & Steroid hormone biosynthesis & $\begin{array}{l}\text { path:00140_25; path:00140_5; path:00140_10; } \\
\text { path:00140_4 }\end{array}$ \\
\hline Pyrvinium & Steroid hormone biosynthesis & $\begin{array}{l}\text { path:00140_6; path:00140_16; path:00140_19; } \\
\text { path:00140_17; path:00140_18; path:00140_4 }\end{array}$ \\
\hline Scriptaid & Steroid hormone biosynthesis & $\begin{array}{l}\text { path:00140_9; path:00140_6; path:00140_17; } \\
\text { path:00140_16; path:00140_5; path:00140_1 }\end{array}$ \\
\hline Trichostatin A & Steroid hormone biosynthesis & $\begin{array}{l}\text { path:00140_10; path:00140_19; path:00140_6; } \\
\text { path:00140_8; path:00140_9 }\end{array}$ \\
\hline $0173570-0000$ & Steroid hormone biosynthesis & $\begin{array}{l}\text { path:00140_16; path:00140_4; path:00140_17; } \\
\text { path:00140_3; path:00140_6; path:00140_10; } \\
\text { path:00140_18; path:00140_13; path:00140_7; } \\
\text { path:00140_8 }\end{array}$ \\
\hline Troglitazone & Cell cycle & path:04110_17 \\
\hline Prochlorperazine & Protein processing in endoplasmic reticulum & path:04141_1 \\
\hline LY-294002 & Steroid hormone biosynthesis & path:00140_6; path:00140_27 \\
\hline Tanespimycin & MAPK signaling pathway & path:04010_15 \\
\hline Monorden & Steroid hormone biosynthesis & path:00140_3; path:00140_7; path:00140_18 \\
\hline
\end{tabular}

CMap, connectivity map.

cells, thus, inhibits the proliferation of tumor cells (35). It was found in vivo that dipalmitoyl phosphatidic acid could dramatically inhibit the growth of tumors in a mouse subcutaneous tumor model, and suppress cell proliferation and 


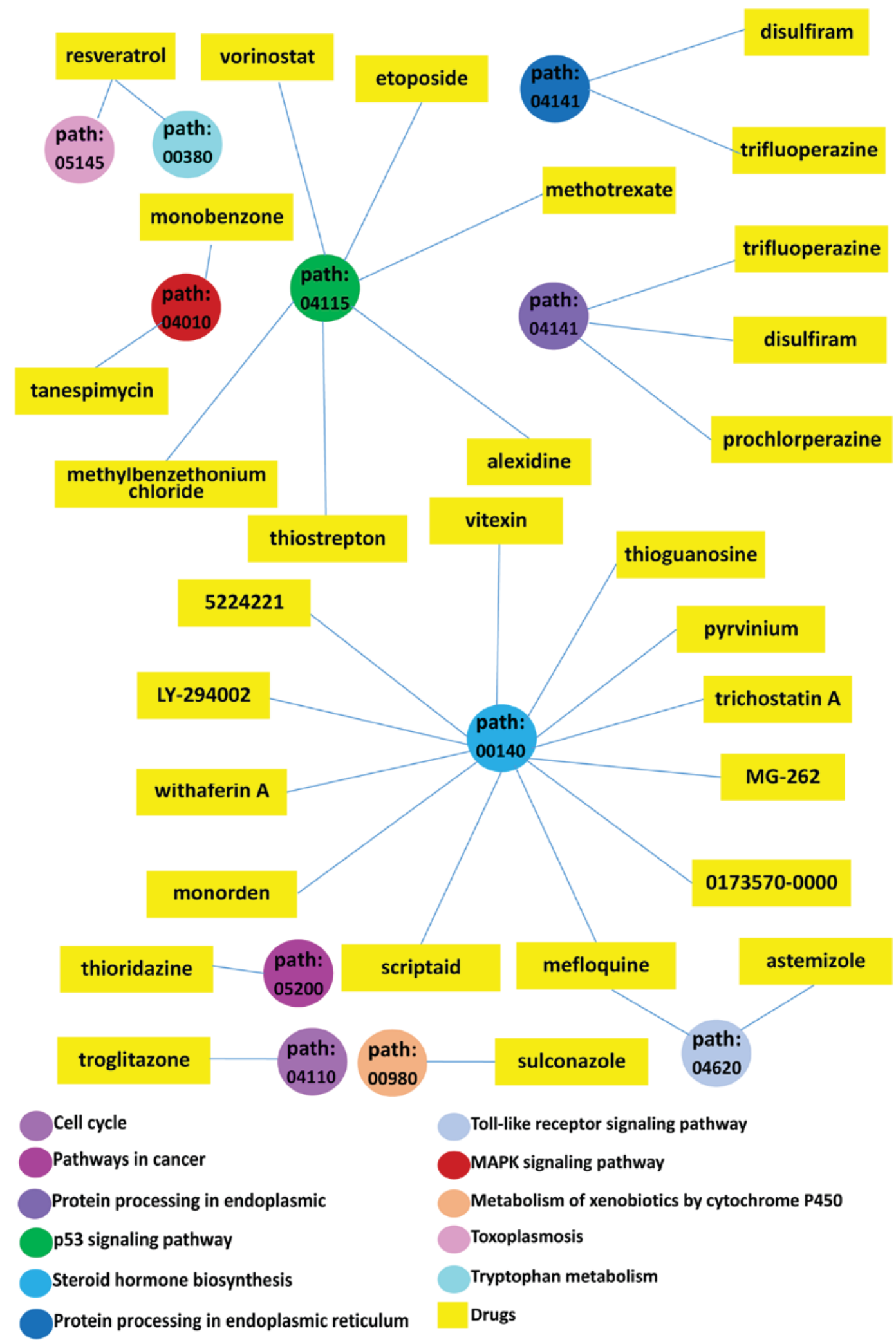

Figure 5. Small-molecular drugs and their perturbed pathways in gastric adenocarcinoma.

angiogenesis in triple-negative breast cancer. The suppressing effect was mediated partly due to reduction in the expression of CCNB1 (36). Therefore, CCNB1 may be an important target gene in the treatment of GAC, and the present study predicted that compounds aimed at this target gene may be reasonable and effective in treating GAC. Recent studies have shown that knockdown of CDC6 expression levels can interfere with the cell cycle and inhibit the proliferation of prostate and ovarian cancer cells $(37,38)$. This evidence suggests that CDC6 may also be a potential biomarker for GAC therapy.

The present study comprehensively analyzed the possible mechanism of treating GAC by data mining in the public gene 
A

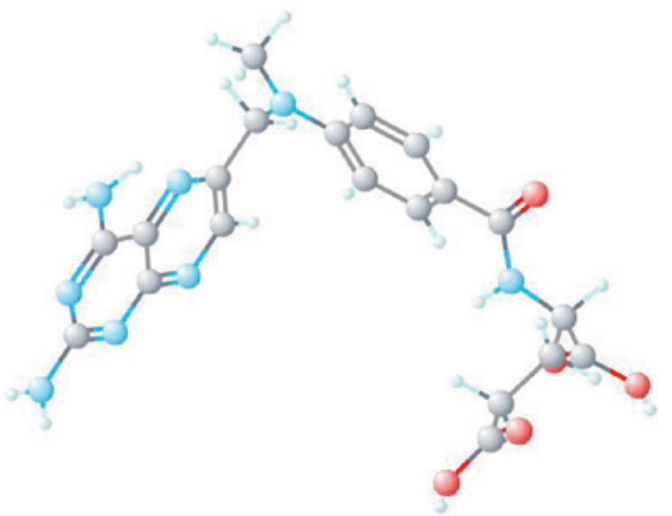

B

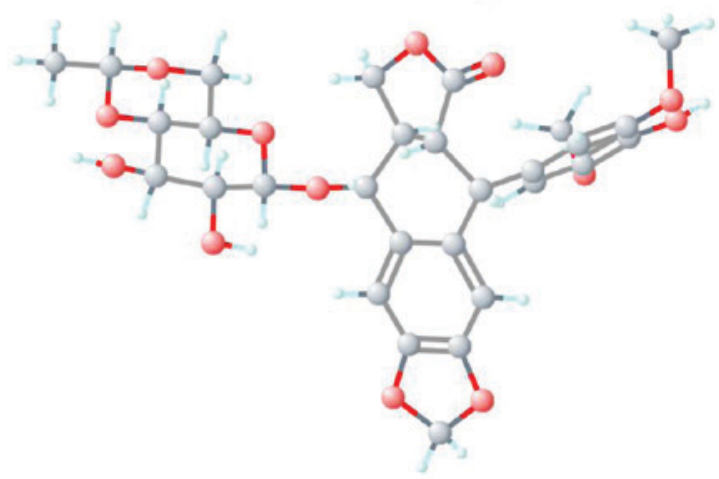

C

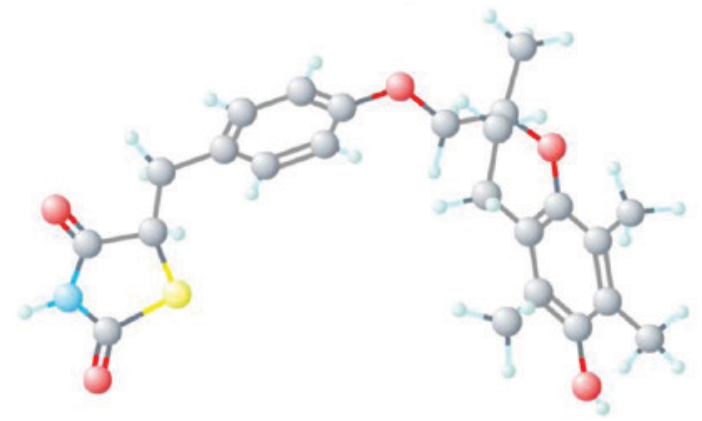

D

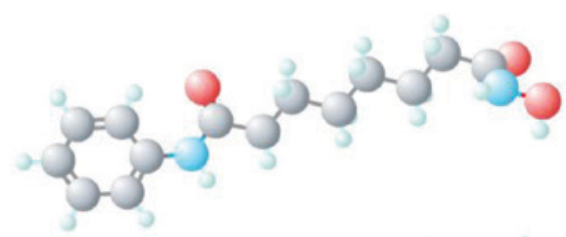

$\mathrm{E}$

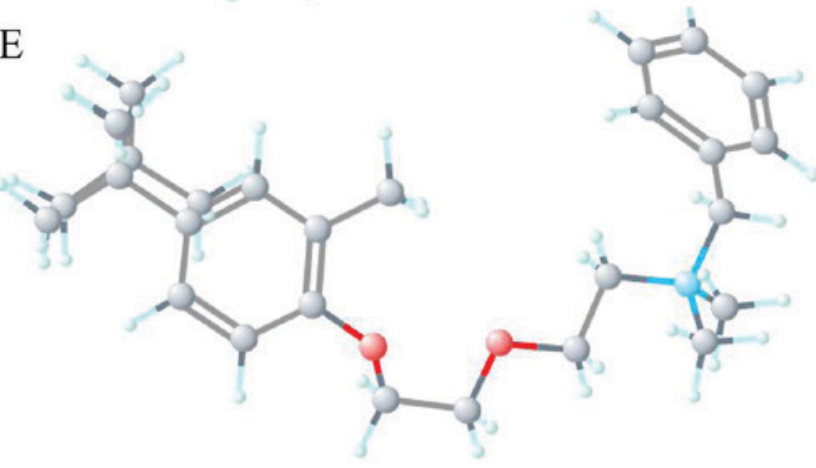

\section{Methotrexate}

Molecular Formula: $\mathrm{C}_{20} \mathrm{H}_{22} \mathrm{~N}_{8} \mathrm{O}_{5}$

PubChem CID: 126941

\section{Etoposide}

Molecular Formula: $\mathrm{C}_{29} \mathrm{H}_{32} \mathrm{O}_{13}$

PubChem CID: 36462

\section{Troglitazone}

Molecular Formula: $\mathrm{C}_{24} \mathrm{H}_{27} \mathrm{NO}_{5} \mathrm{~S}$

PubChem CID: 5591

\section{Vorinostat}

Molecular Formula: $\mathrm{C}_{14} \mathrm{H}_{20} \mathrm{~N}_{2} \mathrm{O}_{3}$

PubChem CID: 5311

Methylbenzethonium Chloride

Molecular Formula: $\mathrm{C}_{28} \mathrm{H}_{44} \mathrm{C}_{1} \mathrm{NO}_{2}$

PubChem CID: 5702238

Figure 6. The 3D conformers of the five compounds that counteracted the molecular signature effect in gastric adenocarcinoma. The 3D structures of the five compounds were provided by PubChem (https://pubchem.ncbi.nlm.nih.gov/compound). (A) Methotrexate, (B) etoposide, (C) troglitazone, (D) vorinostat and (E) methylbenzethonium chloride.

chip databases and bioinformatic analyses. We discovered cell cycle and p53 signaling pathways and key gene targets CCNB1 and CDC6 as potential targets of GAC treatment. We further predicted that seven known compounds may be effective in 

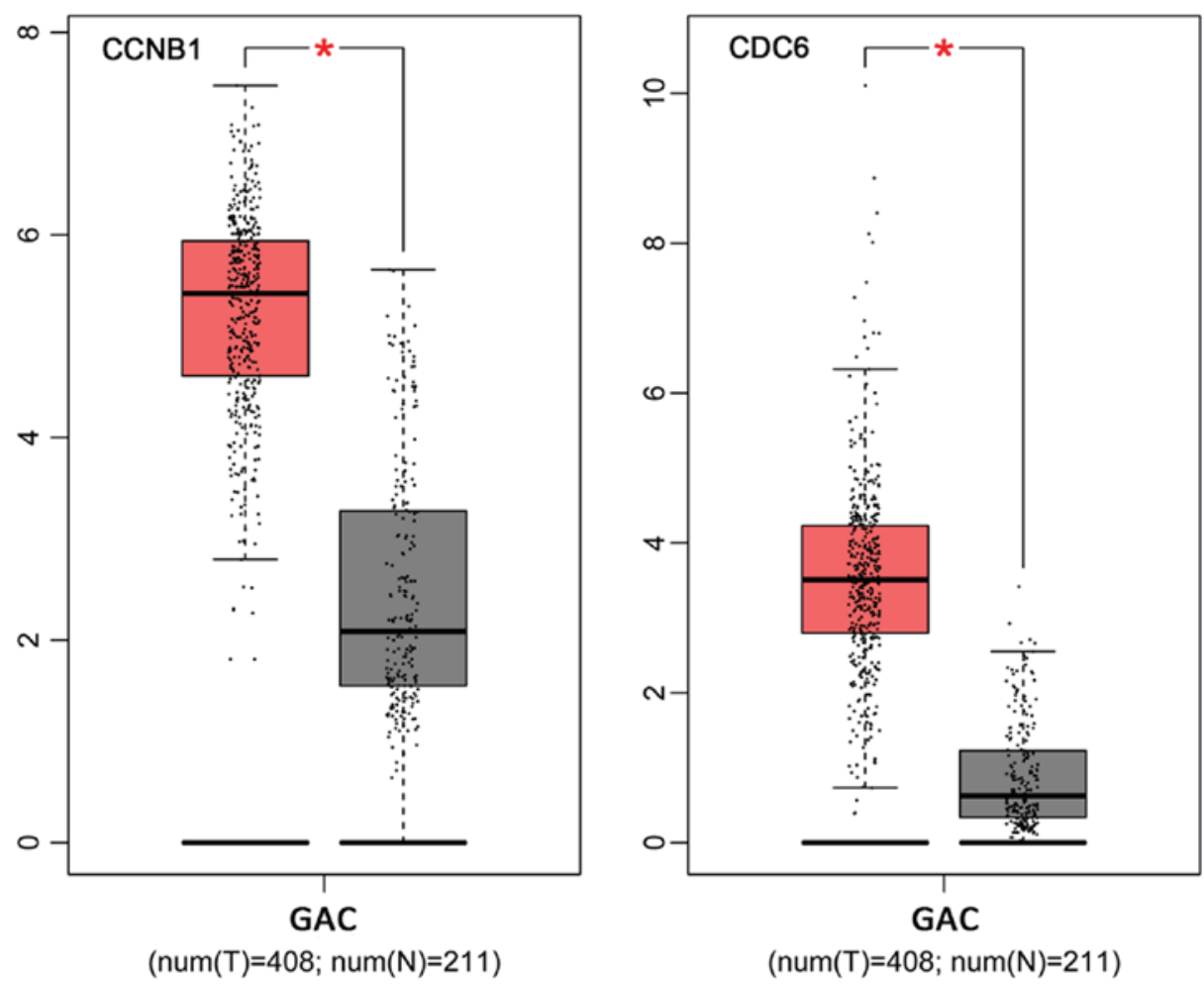

Figure 7. Verification of CCNB1 and CDC6 mRNA expression levels. The data were provided by GEPIA database based on 408 GAC (T; red) and 211 controls (N; grey). GAC, gastric adenocarcinoma, ${ }^{*} \mathrm{P}<0.05$.

curing GAC, including methylbenzethonium chloride and alexidine, which have never been previously reported to treat GAC. However, several limitations should be admitted. Firstly, the current findings were based on in silico methods and validations are certainly needed. Secondly, CMap did not cover GAC cell lines and only provided general DEGs post treatment of existing drugs. The overlapping pathways of DEGs from TCGA and pathways from Cmap also need to be confirmed. Thirdly, the precise mechanism of the drugs we recommended remains to be investigated. Hence, further clinical, in vitro and in vivo experiments are needed to verify the definite effects and molecular mechanism of the potential drugs on GAC.

\section{Acknowledgements}

Not applicable.

\section{Funding}

The present study was supported by a fund from the Promoting Project of Basic Capacity for Young and Middle-Aged University Teachers in Guangxi, China (KY2016YB077).

\section{Availability of data and materials}

The datasets used during the present study are available from the corresponding author upon reasonable request.

\section{Authors' contributions}

ZXC, XPZ, HQY, RZ and JSP analyzed and interpreted the data and wrote the draft of the manuscript. XGQ, RQH,
JM, ZBF, GC and TQG conceived and designed the study, supervised the data mining, corrected and revised the draft. All authors read and approved the manuscript and agree to be accountable for all aspects of the research in ensuring that the accuracy or integrity of any part of the work are appropriately investigated and resolved.

\section{Ethics approval and consent to participate}

Not applicable.

\section{Patient consent for publication}

Not applicable.

\section{Competing interests}

The authors declare that they have no competing interests.

\section{References}

1. Wippel HH, Santos MDM, Clasen MA, Kurt LU, Nogueira FCS, Carvalho CE, McCormick TM, Neto GPB, Alves LR, da Gloria da Costa Carvalho M, et al: Comparing intestinal versus diffuse gastric cancer using a PEFF-oriented proteomic pipeline. J Proteomics 171: 63-72, 2018.

2. Li W, Song D, Li H, Liang L, Zhao N and Liu T: Reduction in peripheral CD19+CD24hCD27+ B cell frequency predicts favourable clinical course in XELOX-treated patients with advanced gastric cancer. Cell Physiol Biochem 41: 2045-2052, 2017.

3. Chen W, Zheng R, Baade PD, Zhang S, Zeng H, Bray F, Jemal A, Yu XQ and He J: Cancer statistics in China, 2015. CA Cancer J Clin 66: 115-132, 2016.

4. Costa NR, Gil da Costa RM and Medeiros R: A viral map of gastrointestinal cancers. Life Sci 199: 188-200, 2018. 
5. Coburn N, Cosby R, Klein L, Knight G, Malthaner R, Mamazza J, Mercer CD and Ringash J: Staging and surgical approaches in gastric cancer: A systematic review. Cancer Treat Rev 63: 104-115, 2018

6. Chen W, Zhou H, Ye L and Zhan B: Overexpression of SULT2B1b promotes angiogenesis in human gastric cancer. Cell Physiol Biochem 38: 1040-1054, 2016.

7. Van Cutsem E, Sagaert X, Topal B, Haustermans K and Prenen H: Gastric cancer. Lancet 388: 2654-2664, 2016

8. Zheng H, Hong H, Zhang L, Cai X, Hu M, Cai Y, Zhou B, Lin J, Zhao $\mathrm{C}$ and $\mathrm{Hu} \mathrm{W}$ : Nifuratel, a novel STAT3 inhibitor with potent activity against human gastric cancer cells. Cancer Manag Res 9: 565-572, 2017.

9. Pavlakis N, Sjoquist KM, Martin AJ, Tsobanis E, Yip S, Kang YK, Bang YJ, Alcindor T, O'Callaghan CJ, Burnell MJ, et al: Regorafenib for the treatment of advanced gastric cancer (INTEGRATE): A multinational placebo-controlled phase II trial. J Clin Oncol 34: 2728-2735, 2016.

10. Belizário JE, Sangiuliano BA, Perez-Sosa M, Neyra JM and Moreira DF: Using pharmacogenomic databases for discovering patient-target genes and small molecule candidates to cancer therapy. Front Pharmacol 7: 312, 2016.

11. Liang L, Zeng JH, Wang JY, He RQ, Ma J, Chen G, Cai XY and Hu XH: Down-regulation of miR-26a-5p in hepatocellular carcinoma: A qRT-PCR and bioinformatics study. Pathol Res Pract 213: 1494-1509, 2017.

12. Xiong DD, Lv J, Wei KL, Feng ZB, Chen JT, Liu KC, Chen G and Luo DZ: A nine-miRNA signature as a potential diagnostic marker for breast carcinoma: An integrated study of 1,110 cases. Oncol Rep 37: 3297-3304, 2017.

13. Zhang Y, Huang JC, Cai KT, Yu XB, Chen YR, Pan WY, He ZL, Lv J, Feng ZB and Chen G: Long non-coding RNA HOTTIP promotes hepatocellular carcinoma tumorigenesis and development: A comprehensive investigation based on bioinformatics, qRT-PCR and meta-analysis of 393 cases. Int J Oncol 51: 1705-1721, 2017.

14. Zhang Y, Dang YW, Wang X, Yang X, Zhang R, Lv ZL and Chen G: Comprehensive analysis of long non-coding RNA PVT1 gene interaction regulatory network in hepatocellular carcinoma using gene microarray and bioinformatics. Am J Transl Res 9: 3904-3917, 2017

15. He RQ, Li XJ, Liang L, Xie Y, Luo DZ, Ma J, Peng ZG, Hu XH and Chen G: The suppressive role of miR-542-5p in NSCLC: The evidence from clinical data and in vivo validation using a chick chorioallantoic membrane model. BMC Cancer 17: 655, 2017.

16. Gao L, Li SH, Tian YX, Zhu QQ, Chen G, Pang YY and Hu XH: Role of downregulated miR-133a-3p expression in bladder cancer: A bioinformatics study. Onco Targets Ther 10: 3667-3683, 2017.

17. Dang YW, Lin P, Liu LM, He RQ, Zhang LJ, Peng ZG, Li XJ and Chen G: In silico analysis of the potential mechanism of telocinobufagin on breast cancer MCF-7 cells. Pathol Res Pract 214: 631-643, 2018

18. Wang J, Li M, Wang Y and Liu X: Integrating subpathway analysis to identify candidate agents for hepatocellular carcinoma Onco Targets Ther 9: 1221-1230, 2016.

19. Musa A, Ghoraie LS, Zhang SD, Glazko G, Yli-Harja O, Dehmer M, Haibe-Kains B and Emmert-Streib F: A review of connectivity map and computational approaches in pharmacogenomics. Brief Bioinform 19: 506-523, 2018.

20. BrumAM,vandePeppelJ,NguyenL,AlievA,Schreuders-KoedamM, Gajadien T, van der Leije CS, van Kerkwijk A, Eijken M, van Leeuwen JPTM and van der Eerden BCJ: Using the connectivity map to discover compounds influencing human osteoblast differentiation. J Cell Physiol 233: 4895-4906, 2018.

21. Busby J, Murray L, Mills K, Zhang SD, Liberante F and Cardwell CR: A combined connectivity mapping and pharmacoepidemiology approach to identify existing medications with breast cancer causing or preventing properties. Pharmacoepidemiol Drug Saf 27: 78-86, 2018.

22. Xiao SJ, Zhu XC, Deng H, Zhou WP, Yang WY, Yuan LK, Zhang JY, Tian S, Xu L, Zhang L and Xia HM: Gene expression profiling coupled with connectivity map database mining reveals potential therapeutic drugs for Hirschsprung disease. J Pediatr Surg 53: 1716-1721, 2018.
23. Varadan P, Ganesh A, Konindala R, Nagendrababu V, Ashok R and Deivanayagam K: Comparison of the antibacterial efficacy of alexidine and chlorhexidine against enterococcus faecalis: An in vitro study. Cureus 9: e1805, 2017

24. Feng R, Rios JA, Onishi T, Lokshin A, Gorelik E and Lentzsch S: Cell-based and cytokine-directed chemical screen to identify potential anti-multiple myeloma agents. Leuk Res 34: 917-924, 2010.

25. Conesa C, Doss MX, Antzelevitch C, Sachinidis A, Sancho J and Carrodeguas JA: Identification of specific pluripotent stem cell death-inducing small molecules by chemical screening. Stem Cell Rev 8: 116-127, 2012

26. Huang H, Han Y, Yang X, Li M, Zhu R, Hu J, Zhang X, Wei R, Li K and Gao R: HNRNPK inhibits gastric cancer cell proliferation through $\mathrm{p} 53 / \mathrm{p} 21 / \mathrm{CCND} 1$ pathway. Oncotarget 8: 103364-103374, 2017.

27. Fu H, Wang C, Yang D, Wei Z, Xu J, Hu Z, Zhang Y, Wang W, Yan $\mathrm{R}$ and Cai Q: Curcumin regulates proliferation, autophagy, and apoptosis in gastric cancer cells by affecting PI3K and P53 signaling. J Cell Physiol 233: 4634-4642, 2018.

28. Sun M, Si G, Sun HS and Si FC: Inhibition of CREPT restrains gastric cancer growth by regulation of cycle arrest, migration and apoptosis via ROS-regulated p53 pathway. Biochem Biophys Res Commun 496: 1183-1190, 2018.

29. Wang C, Wang J and Bai P: Troglitazone induces apoptosis in gastric cancer cells through the NAG-1 pathway. Mol Med Rep 4: 93-97, 2011.

30. Li X, Qiu W, Liu B, Yao R, Liu S, Yao Y and Liang J: Forkhead box transcription factor 1 expression in gastric cancer: FOXM1 is a poor prognostic factor and mediates resistance to docetaxel. J Transl Med 11: 204, 2013.

31. Yoo C, Ryu MH, Na YS, Ryoo BY, Lee CW, Maeng J, Kim SY, Koo DH, Park I and Kang YK: Phase I and pharmacodynamic study of vorinostat combined with capecitabine and cisplatin as first-line chemotherapy in advanced gastric cancer. Invest New Drugs 32: 271-278, 2014.

32. Ebrahimifar M, Hasanzadegan Roudsari M, Kazemi SM, Ebrahimi Shahmabadi H, Kanaani L, Alavi SA and Izadi Vasfi M: Enhancing effects of curcumin on cytotoxicity of paclitaxel, methotrexate and vincristine in gastric cancer cells. Asian Pac J Cancer Prev 18: 65-68, 2017.

33. Duo-Ji MM, Ci-Ren BS, Long ZW, Zhang XH and Luo DL: Short-term efficacy of different chemotherapy regimens in the treatment of advanced gastric cancer: A network meta-analysis. Oncotarget 8: 37896-37911, 2017.

34. Shi Q, Wang W, Jia Z, Chen P, Ma K and Zhou C: ISL1, a novel regulator of CCNB1, CCNB2 and c-MYC genes, promotes gastric cancer cell proliferation and tumor growth. Oncotarget 7 : 36489-36500, 2016.

35. Li Y, Ji S, Fu LY, Jiang T, Wu D and Meng FD: Knockdown of cyclin-dependent kinase inhibitor 3 inhibits proliferation and invasion in human gastric cancer cells. Oncol Res 25: 721-731, 2017.

36. Zhang QQ, Chen J, Zhou DL, Duan YF, Qi CL, Li JC, He XD, Zhang M, Yang YX and Wang L: Dipalmitoylphosphatidic acid inhibits tumor growth in triple-negative breast cancer. Int J Biol Sci 13: 471-479, 2017.

37. Karanika S, Karantanos T, Li L, Wang J, Park S, Yang G, Zuo X, Song JH, Maity SN, Manyam GC, et al: Targeting DNA damage response in prostate cancer by inhibiting androgen receptor-CDC6-ATR-Chk1 signaling. Cell Rep 18: 1970-1981, 2017.

38. Deng Y, Jiang L, Wang Y, Xi Q, Zhong J, Liu J, Yang S, Liu R, Wang J, Huang M, et al: High expression of CDC6 is associated with accelerated cell proliferation and poor prognosis of epithelial ovarian cancer. Pathol Res Pract 212: 239-246, 2016.

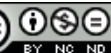

This work is licensed under a Creative Commons Attribution-NonCommercial-NoDerivatives 4.0 International (CC BY-NC-ND 4.0) License. 